\title{
RESPONSABILIDAD SOCIAL DE LA UNIVERSIDAD: UN SOL DE ESPERANZA PARA EL NEA
}

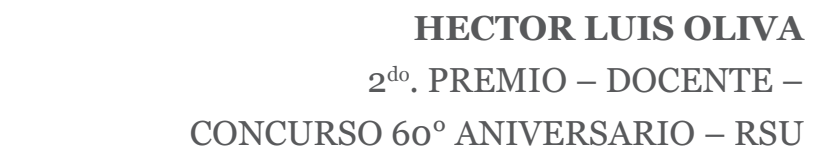

FACULTAD DE CIENCIAS ECONÓMICAS - UNNE 


\section{INTRODUCCION}

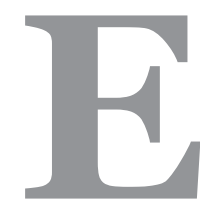

l proceso de globalización ha incidido fuertemente en la redefinición de fronteras económicas y ha tenido un impacto significativo en el alcance de las acciones públicas y privadas. Las decisiones políticas de los gobiernos ya no pueden ignorar las condiciones cambiantes que genera el nuevo orden económico mundial basado en la apertura comercial; y el sector privado tampoco puede hacer caso omiso de las nuevas demandas.

El mundo cada vez más competitivo obliga a los sectores públicos y privados a poner más atención en la promoción de condiciones que mejoren la competitividad, la educación, el desarrollo de competencias y capacidades innovadoras dentro de la fuerza laboral, la salud, la reducción de riesgos y vulnerabilidades, así como la preservación del medio ambiente.

Bernardo Kliksberg, afirma que "la globalización ha tenido lugar en un vacío ético, donde el éxito y los fracasos del mercado tendían a convertirse en el máximo estándar de conducta”. En la actualidad, la discusión sobre la ética es impulsada por la ciudadanía, quienes demandan una respuesta efectiva como consumidores y como clientes de las empresas (Kliksberg, 2008:11).

Como consecuencia de esa nueva visión empresarial; en los últimos años hay una tendencia hacia un mayor compromiso de las organizaciones en general,- incluyendo a las universidades- por asumir una mayor responsabilidad social, no sólo en su ambiente interno sino también en relación con su entorno.

De acuerdo con la Declaración Mundial de la UNESCO sobre Educación Superior en el siglo XXI, los dos principales propósitos académicos de la universidad son la formación humana y profesional y la construcción de nuevos conocimientos.

Para atender esos propósitos, es menester que las universidades asuman la Responsabilidad Social Universitaria como un compromiso de gestión y difusión del conocimiento, e inicien un proceso de cambio y transformación organizacional inclusiva, innovadora y creativa.

En virtud de lo anteriormente indicado, el presente documento tiene como propósito aportar a la discusión y reflexión sobre la responsabilidad social universitaria, y despertar el interés para iniciar la transformación hacia una gestión comprometida con el capital social y el desarrollo humano sostenible.

En primer lugar señalamos los desafíos éticos de nuestro tiempo, luego incursionamos en las responsabilidades que deben asumir los diferentes actores sociales para el desarrollo ético y posteriormente analizamos el rol que le corresponde a la Universidad Nacional del Nordeste en este siglo, pleno en oportunidades y contradicciones.

\section{LA ETICA EN EDUCACION SUPERIOR}

El sentido más antiguo de la ética (de origen griego) residía en el concepto de la morada o lugar donde se habita; luego referido al hombre o pueblos se aplicó en el sentido de su país, tomando especial prestigio la definición utilizada por Heidegger: "es el pensar que afirma la 
morada del hombre", es decir su referencia original, construida al interior de la íntima complicidad del alma. En otras palabras ya no se trataba de un lugar exterior, sino del lugar que el hombre porta a sí mismo. "El ethos" es el suelo firme, el fundamento de la praxis, la raíz de la que brotan todos los actos humanos. Lo ético comprende la disposición del hombre en la vida, su carácter, costumbre y moral. Podríamos traducirla como "el modo o forma de vida" en el sentido profundo de su significado. La ética florece a partir de nuestros valores que nos dictan si algo esta bien o mal (correcto o incorrecto) en un acto humano.

La ética es la disciplina del conocimiento que estudia las actitudes, hábitos y costumbres del ser humano. Estudia y explica el porqué de la conducta humana y propone formas deseables para actuar."La ética es la morada o lugar donde se habita, es el refugio de toda persona, la fortaleza inexpugnable del ser humano. El suelo firme para caminar sobre la vida. La ética es una lámpara que ilumina al hombre en su vida"1.

La Universidad es un bien social. Es decir, ella ha surgido y se explica en función de la sociedad.

La Universidad es para ella y, en ese sentido, le pertenece: no es un bien privado sino social. En consecuencia, la Universidad no sólo debe actuar teniendo como meta el bien de la sociedad, sino que debe darle cuenta a ella de su gestión.

Toda labor académica de la Universidad y de sus unidades tiene un contenido altamente social. Ella busca, en efecto, formar hombres y mujeres integrales que presten un servicio profesional altamente capacitado a la sociedad, con sólidos criterios éticos y humanísticos de solidaridad y excelencia en el servicio.

La investigación en la Universidad pretende desarrollar un conocimiento, tanto científico como técnico, que permita una mejor y mayor calidad de vida. Y ello es altamente social.

Pero más allá de esa contribución genérica, debe haber una contribución específica y particular que permita responder a la situación concreta de la sociedad en que vive. La Universidad y sus unidades no pueden ser ajenas a su contorno; deben no sólo situarse, sino orientarse hacia él y de alguna manera dejarse conformar por sus necesidades y urgencias.

Como resultado de lo anterior, la Universidad debe extremarse en la búsqueda y constitución de ciencias y técnicas pertinentes, es decir, de ciencias que toquen la realidad del país o de la región para ayudar a transformarla de manera inteligente y eficaz.

En la declaración mundial sobre la educación superior del siglo XXI, la pertinencia social; se la menciona como "un importante referente teórico que ha permitido orientar las concepciones de responsabilidad social universitaria”; la pertinencia de la educación superior debe evaluarse en función de la adecuación entre lo que la sociedad espera de las instituciones y lo que estas hacen.

La Universidad no puede sustraerse de la lucha contra la pobreza, la exclusión social, el desempleo, el analfabetismo y el acceso a la salud y la educación; que son de naturaleza ética, porque hacen a valores centrales para el sentido mismo de la vida.

Pobreza que mata, familias destruidas, niños indefensos, mujeres discriminadas, pue- 
blos indígenas marginados, desigualdades extremas, no son temas adicionales de la agenda macroeconómica nacional ni regional. Son desafíos éticos ineludibles, que están amenazando el derecho más básico, el derecho a la vida de amplios sectores de la población de nuestro país.

Un prominente pensador, el Rabino Abraham Yoshua Heschel, decía que hacernos responsables por los otros no merece ningún premio, ni distinción, es simplemente "la manera de vivir correcto" 2 .

Una parte del pensamiento económico de más circulación ha sugerido implícitamente la necesidad de separar ética y economía. La economía seria un área a manejar por técnicos experimentados, y la ética pertenecería a otra esfera esencialmente espiritual, y no debería interferir.

La ética debería orientar y regular la economía. Lo exigía Juan Pablo II planteando que: "una economía que no tenga en cuenta la dimensión ética no puede llamarse a si misma una economía en el sentido de un uso racional y constructivo de la riqueza material”3.

La superación de la escisión entre ética y economía abre la agenda de las responsabilidades de los principales actores sociales, como el Estado, la empresa, la universidad y la sociedad civil en todas sus expresiones. La asunción de responsabilidades sociales por parte de todos ellos, y la acción coordinada pueden hacer diferencias muy relevantes.

La Universidad como pilar de la investigación, la transferencia de conocimientos, y la preparación de las nuevas generaciones de profesionales, no puede conformarse con "rendimientos mediocres".

Debe auto-exigirse y pedir a todos sus estamentos, conformar equipos que marchen en dirección de las nuevas fronteras tecnológicas; y deben enlazarse activamente con los sectores productivos.

Pero junto con su rol científico y tecnológico, y su gran importancia como centro de cultura, la Universidad tiene en este siglo una responsabilidad social clave que debe asumir en plenitud. Tiene que estar en la avanzada de la lucha por enfrentar los desafíos éticos de nuestro tiempo. No puede ser una entidad más en ese esfuerzo, ni seguir a los otros actores, debe marcar caminos.

Esta responsabilidad social debe materializarse en políticas concretas con expresión institucional.

Entre ellas, el de la formación ética de los jóvenes que acuden a la institución de educación superior, garantizarles una preparación profesional de la mejor calidad; de excelencia.

La Universidad debe preparar economistas, administradores, médicos, abogados, ingenieros, y especialistas de todos los campos, que tengan una sólida base de responsabilidad, para decidir en base a valores morales fundamentales, en los dilemas éticos que se les planteen, y asuman compromisos de servicio con los más débiles.

"El papel que realiza la educación en las universidades, es fundamental para que las nuevas generaciones de profesionales encuentren, reconozcan y asuman sus responsabilidades éticas" (Kliksberg, 2004).

\footnotetext{
${ }^{2}$ Heschel, Abraham Yoshua (1959). Dios en busca del hombre. Meridien libros y la publicación judía. Sociedad para Estados Unidos.

3 Juan Pablo II (2000). Mensaje para la celebración del Día Mundial de la Paz.
} 
Esa preparación requiere trabajar no sólo con textos, sino con experiencias concretas, y contacto activo con la realidad. El ideal no es dictar una materia de ética en el currículum, sino transversalizar la enseñanza de la ética aplicada, trabajando en cada área los problemas éticos propios de la misma, y el compromiso con la construcción de un mundo solidario.

La responsabilidad social debe estar presente en la agenda de investigación, priorizando temáticas que representan los problemas más apremiantes de la población.

En las sociedades democráticas la formación de las políticas públicas que se adopten esta fuertemente influida por el debates sobre cuestiones como; entre muchas otras; la creación de trabajo, la discriminación de género, la exclusión social, las causas de la criminalidad, y las inequidades. El aporte de la Universidad desde la investigación seria, y la voz de la ciencia, puede aportar mucho al mejoramiento de la calidad del debate, y la visualización de alternativas de decisión que no sean "más de lo mismo".

Debe extender su acción educativa a la sociedad en su conjunto mediante una tarea sistemática que coloque su potencial docente y de investigación al servicio de empresas, sindicatos, organizaciones de la sociedad civil, y muchas otras instituciones.

Especialmente importante es la labor que puede hacer capacitando y fortaleciendo a los desfavorecidos.

La Universidad integrada por jóvenes que tienen un potencial de voluntariado muy importante; debe promover y apoyar la puesta en marcha de ese potencial. Ello tendrá efectos educativos de primer orden, y puede ser una fuente de aportes muy significativos a las mejores causas.

Es importante rechazar el uso común de la expresión "Responsabilidad Social" como sinónima de "generosidad social", "beneficencia social" o "iniciativa de solidaridad". La expresión de la solidaridad y caridad humana se suele llamar filantropía.

La Responsabilidad Social es una teoría de gestión que obliga a la organización a situarse y comprometerse socialmente en y desde el mismo ejercicio de sus funciones básicas, no es la expresión de su solidaridad filantrópica, es la filosofía de gestión que practica a diario en todos sus ámbitos de competencia, desde su administración central, para garantizar que no entre en contradicción con sus deberes para con la sociedad.

Distinguiendo la Responsabilidad Social de la filantropía, no estamos diciendo que no hay que practicar la filantropía. Es un valor ético de primera importancia y de alta pertinencia formativa que deberíamos promover en todas las entidades educativas. Pero una organización que practica la filantropía no por eso puede autoproclamarse como socialmente responsable.

A la labor política de la Universidad corresponde el generar una nueva Ética y unos nuevos valores. El bien común no podrá realizarse si no es sobre la base de principios que garanticen la humanidad del hombre y de sus relaciones (ética) así como la dinámica afectiva y emocional (valores) que mueva a la prosecución del bien de todos, por encima del egoísmo y de los intereses particulares de individuos o grupos. 


\section{DESARROLLO ETICO}

Las políticas públicas que en una sociedad democráticas encarnan la acción colectiva; deben tener como prioridad el garantizar a todos los ciudadanos junto a las libertades públicas, sus derechos básicos a educación, salud, trabajo, vivienda, posibilidad de mantener a su familia.

En 1989 la Asamblea General de la ONU había sancionado por unanimidad "el derecho al desarrollo”, y había estipulado que los primeros responsables de asegurarlo eran los gobiernos.

Durante muchos años, el debate en torno a las políticas sociales en nuestro país se concentraba en dos posturas: por un lado, quienes consideraban que el Estado tenía como tarea principal asistir y garantizar el acceso de los bienes básicos a la población más vulnerable y, por el otro, aquellos que consideraban que la política social debía generar capacidades para que la gente pudiera mejorar su condición social.

Las decisiones económicas tienen a diario impactos enormes en las condiciones de vida cotidiana de grandes sectores; se debe superar la inestabilidad y la tecnocratización y tener en cuenta el objetivo final de la economía: el bienestar de la gente.

Este esquema ha tenido serias dificultades y Argentina se encuentra frente a una situación social de marginación y exclusión social, con el desafío de generar el capital humano (las capacidades), el capital social (las condiciones para asociarse) y complementarlo con el capital económico, es decir, con el financiamiento de actividades productivas que generen trabajo e inclusión social.

El movimiento de la Responsabilidad Social está en plena efervescencia, dentro del más amplio movimiento de la promoción del "desarrollo sostenible (o sustentable)", y evoluciona sobre el trasfondo de la toma de conciencia mundial de que el modelo actual de desarrollo es insostenible para el planeta, tanto a nivel ambiental como social.

Para ello, es importante generar condiciones para fortalecer los grupos sociales así como identificar nichos productivos reales en cada región del país.

Procurar el desarrollo productivo como prioridad para la política social significa tener la capacidad para articular en cada territorio las perspectivas de un crecimiento económico sustentable.

En este marco, es menester redoblar esfuerzos por parte de todos los sectores de la comunidad para consolidar el crecimiento económico y la distribución equitativa de los recursos, con estrategias institucionales que favorezcan los grandes consensos y la institucionalización de las políticas de Estado.

De esta manera, es significativo destacar la responsabilidad social de los sectores que se encuentran vinculados directa e indirectamente a las políticas públicas, entre estos la Universidad.

En un mundo que puede, pero que no hace lo necesario, ha llegado la hora de que cada actor social asuma sus responsabilidades sociales. Juan Pablo II (2000) invitó: "a los economistas y líderes profesionales financieros, así como a los líderes políticos a reconocer la urgencia de asegurar que las practicas económicas y que las políticas vinculadas tengan como meta el bien de cada persona, y de la personas en su totalidad".

La Responsabilidad Social Universitaria es una nueva filosofía de gestión universitaria que pretende renovar el compromiso social de la Universidad al mismo tiempo que facilitar 
soluciones innovadoras a los retos que enfrenta la educación superior en el contexto de un mundo globalizado pero insostenible en sus patrones de "desarrollo".

Bernardo Kliksberg (2004), profundiza más sobre este aspecto indicando que los especialistas en ciencias gerenciales deberían ser formados para impulsar un avance en las prácticas de responsabilidad social empresarial; en tanto los contadores deberían velar por la protección de los intereses de la comunidad garantizando confiabilidad y transparencia total en la información tanto en el área pública como privada. Cabe agregar que los economistas deberían contribuir en la generación de una economía que enfrente las tremendas exclusiones actuales.

En base a ello las Universidades comprenden al rol de extensión como factor de interacción entre la actividad de Investigación y desarrollo de la Universidad y la Comunidad; siendo que a través de esta actividad académica, la universidad comparte sus riquezas (bienes y saberes) generando un estrecho vínculo con el fenómeno social a fin de producir las transformaciones necesarias para el logro de una mejor calidad de vida.

Para que esta actividad no sea simplemente un acto de dar, sino una actividad de integración y compromiso, la Universidad debe velar por brindar los medios necesarios que permitan la inclusión de todos los sectores de la comunidad y actuar como un nexo entre las diferentes clases sociales. La universidad debe potenciar intelectualmente a las clases marginales, que necesitan del aporte de la universidad para poder crear un ámbito social de convivencia.

La acción política de la Universidad ha de consistir en proyectar su saber sobre la sociedad para transformarla; definiendo su modelo de intervención en la sociedad de manera que pueda actuar en forma coherente, sistemática y ordenada.

La Universidad, debe tener como uno de sus objetivos primordiales el ser factor de desarrollo y transformación de la sociedad en que vive. Debe, por consiguiente, insertarse en la realidad nacional, regional, estudiando, de manera interdisciplinaria, los grandes problemas que vive el país o la región, produciendo conocimientos relevantes sobre estos problemas y presentando estrategias y alternativas de solución que, de una manera seria y responsable, permitan la transformación de la sociedad.

El análisis crítico de nuestro modelo de desarrollo y sus alternativas; el análisis de la situación rural (producción y comercialización de productos, reforma agraria, movimientos rurales, cultivos y modelos de desarrollo alternativo); los recursos naturales y el medio ambiente, el desarrollo sostenible, la política urbana, el flagelo del desempleo, los procesos de socialización y tantos otros. La labor académica y política de la Universidad debe conducir no sólo a diagnósticos, análisis y proyectos, sino a formular cómo realizar dichos proyectos.

Esto constituye un paso de carácter verdaderamente cualitativo: es el paso de la representación y el pensamiento al estadio de la acción; es el paso del "saber" al "saber hacer", de la teoría a la praxis; por ello esta labor resulta absolutamente imprescindible.

La cultura consiste en el cultivo real, activo, racional y científico de la realidad social a través de valores, sentidos y símbolos. El proyecto de una nueva cultura ha de tener como primer objetivo a la Universidad misma en la que el clima o cultura institucional corresponda a los 
valores que explícitamente quiere cultivar: el respeto por el otro, la aceptación de su dignidad y diversidad, la exclusión de cualquier tipo de violencia, aún sutil, la solución pacífica de los conflictos, el acceso a una justicia real, entre otros. De esta cultura institucional deberán ser portadoras todas las personas de la Universidad, no importa su oficio o su jerarquía.

Finalmente, la nueva cultura ha de ofrecer salidas auténticas a las profundas inquietudes espirituales del ser humano, la principal de las cuales se refiere al sentido último de su existencia: ¿Quiénes somos realmente, de dónde venimos y para dónde vamos?

Pero este nuevo proyecto de cultura, ha de tener como objetivo, más allá de la Universidad, la sociedad misma; debe esforzarse por colaborar en conformar la nueva sociedad.

\section{LA UNNE EN EL SIGLO XXI}

Cuando una sociedad como la nuestra padece males que parecen insolubles, la Universidad es especialmente responsable de pensar, de imaginar, de construir utopías, de inventar.

Apasionante reto para el universitario a pensar, a imaginar, a crear perspectivas que contemplen no sólo respuestas a los objetivos problemas económicos, sino respuestas más permanentes al sentido total de una existencia más humana.

Las Universidad es una organización que forma parte del tejido social; es centro del conocimiento; cuya razón de ser es poner el mismo al servicio de la sociedad que la cobija; es actor social clave y no escapa a la exigencia de ejercer la responsabilidad social.

La Universidad Nacional del Nordeste debe tener como uno de sus objetivos propios el de colaborar en la creación de un nuevo proyecto de comunicación social.

La Universidad debe, en primer lugar, esforzarse por conocer científicamente la manera de como se construye la actual opinión pública, sus formas de actuación, movilización y manipulación, con miras a colaborar en la construcción de una opinión pública bien informada y orientada eficazmente a la búsqueda del auténtico bien social.

Los medios de comunicación social deben tomar conciencia que la UNNE como fuerza educadora de la región; es promotora de los más auténticos valores humanos y tiene un importante protagonismo en la construcción de la nueva región del NEA.

Las funciones universitarias de gestión, docencia, investigación y extensión; cuando son ejercidas con una perspectiva ética y al servicio de todos los sectores de la sociedad, contribuyen a poner de manifiesto la responsabilidad social universitaria, de la que deben ser protagonistas todos los estamentos universitarios, principalmente los estudiantes.

Las funciones sustantivas de la universidad han estado signadas por diversos tipos de contradicciones u obstáculos, que no han permitido consolidar, en algunos casos, una verdadera integración y comunicación entre la docencia, la investigación y la extensión.

La función de extensión y la responsabilidad social universitaria se complementan; esta ultima se convierte en un parámetro que define de manera clara el componente ético y dirige la 
gestión de las funciones sustantivas en general hacia objetivos comunes, con una visión compartida por la comunidad universitaria; construyendo directrices que respondan a un modelo con mayor pertinencia social.

Con respecto a la docencia; asumir el paradigma de la responsabilidad social universitaria significa promover estrategias pedagógicas que favorezcan los aprendizajes permanentes y socialmente pertinentes, tales como la conformación de comunidades de aprendizaje para el desarrollo mutuo vinculadas con materias de las carreras, el empleo del aprendizaje basado en problemas y aprendizajes basados en proyectos sociales que permitan al estudiante vivenciar las dificultades reales que aquejan a su localidad o provincia, estrategias que promuevan la multi, inter y transdisciplinaridad humanística, profesional y ética, así como la reflexión crítica sobre las teorías y paradigmas enseñados, sus consecuencias e impactos sociales.

La investigación como actividad sustantiva, también está llamada a transformaciones de fondo para contribuir al desarrollo de la responsabilidad social universitaria. Es necesario impulsar proyectos de investigación realizados bajo el enfoque de varias disciplinas, desarrollar actividades pedagógicas de investigación-acción y estimular la investigación con las demandas sociales, ambientales y económicas más relevantes del entorno, mediante la implementación de proyectos en colaboración con actores no universitarios para la solución de problemas sociales reales, fomentando así la investigación participativa en comunidades de aprendizaje, realizar investigaciones aplicadas al desarrollo humano sustentable, fomentando la gestión social del conocimiento de manera responsable, a fin de garantizar la pertinencia social del conocimiento producido.

También se deben tener en cuenta determinados aspectos de índole organizacional. La Universidad debe promover la responsabilidad social no solo desde sus funciones sustantivas, sino además desde todas sus áreas administrativas y de dirección.

Desde esta perspectiva la organización debe definir y cumplir en forma congruente con su misión organizacional, implementar un código de ética y un comité autónomo encargado de promover y vigilar su cumplimiento, asegurar no sólo el cumplimiento de las leyes nacionales sino el de los más exigentes estándares internacionales (laborales, sociales, ambientales), asegurar un buen clima laboral, luchar contra la discriminación y la inequidad, proteger los derechos humanos de las personas y cumplir con reportar en forma transparente y confiable los resultados del desempeño organizacional, son una serie de recomendaciones que promueven los instrumentos de gestión de la Responsabilidad Social.

La filosofía gerencial de la Responsabilidad Social invita a la Universidad a tomar conciencia de todas las consecuencias y efectos que sus estrategias y actuaciones provocan en los ámbitos humanos, sociales y ambientales.

El camino más práctico para delimitar lo que es el proceso de Responsabilización Social Universitaria pasa por resaltar la definición de la Responsabilidad Social en términos de gestión de impactos. 
Estos pueden ser agrupados en cuatro grandes rubros:

a- Impactos de funcionamiento organizacional: Como cualquier organización laboral, la Universidad genera impactos en la vida de su personal administrativo, docente y estudiantil. La Universidad deja "huellas" en las personas que viven en ella. ¿Cuáles son los valores que vivimos a diario? ¿Cómo debemos vivir en nuestra universidad en forma responsable, en atención a la naturaleza, la dignidad y el bienestar de los miembros de la comunidad universitaria?.

b- Impactos educativos: La Universidad tiene por supuesto un impacto directo sobre la formación de los jóvenes y profesionales, su manera de entender e interpretar el mundo, comportarse en él y valorar ciertas cosas en su vida. Influye asimismo sobre la moralidad profesional, orienta (de modo consciente o no) la definición de la ética profesional de cada disciplina y su rol social. ¿Qué tipo de profesionales y personas venimos formando? ¿Cómo debemos estructurar nuestra enseñanza para formar ciudadanos responsables del desarrollo del país y la región en forma justa y sostenible?.

c- Impactos cognitivos y epistemológicos: La Universidad orienta la producción del saber y las tecnologías, influye en la definición de lo que se llama socialmente "Verdad, Ciencia, Racionalidad, Legitimidad, Utilidad, Enseñanza, etc." Incentiva (o no) la fragmentación y separación de los saberes al participar en la delimitación de los ámbitos de cada especialidad. Articula la relación entre tecnociencia y sociedad, posibilitando (o no) el control y la apropiación social de la ciencia. Genera actitudes como el elitismo científico, la "expertocracia" o al contrario promueve la democratización de la ciencia. Influye finalmente sobre la definición y selección de los problemas de la agenda científica. ¿Qué tipo de conocimientos producimos, para qué y para quiénes? ¿Qué conocimientos debemos producir y cómo debemos difundirlos para atender las carencias cognitivas que perjudican el desarrollo social sostenible del país y la región?.

d- Impactos sociales: La Universidad tiene un impacto sobre la sociedad y su desarrollo económico, social y político. No sólo tiene un impacto directo sobre el futuro del país y la región en cuanto forma a sus profesionales y líderes, sino que ella es también un referente y un actor social, que puede promover (o no) el progreso, que puede crear (o no) capital social, vincular (o no) la educación de los estudiantes con la realidad social exterior, hacer accesible (o no) el conocimiento a todos. Así, el entorno social de la Universidad se hace una cierta idea de su papel y su capacidad (o no) de ser un interlocutor válido y útil en la solución de sus problemas. ¿Qué papel asumimos en el desarrollo de la sociedad, con quiénes y para qué? ¿Cómo la Universidad puede ser, desde su función y pericia específica, un actor partícipe del progreso social a través del fomento de capital social?.

Más allá de las partes interesadas, estrechamente vinculadas con la Universidad, se puede distinguir otros actores sociales más o menos afectados por el desempeño de la Universidad, para tomarlos en cuenta al momento de establecer las áreas de mejora hacia la Responsabilización Social Universitaria. Estos son los padres de familia de los estudiantes, los familiares de los empleados, los empleadores de los futuros profesionales, los egresados, los Colegios profesionales, todos los sectores del Estado que se vinculan con la Universidad (sector educativo, 
ciencia y tecnología, economía, social, ambiental, etc.), hasta las generaciones futuras porque su existencia y bienestar depende sumamente de lo que se enseña hoy en la Universidad a los futuros científicos y líderes políticos de la región y del país.

Estos cuatro impactos definen cuatro ejes de gestión socialmente responsables de la Universidad:

1- Gestión socialmente responsable de la organización misma, del clima laboral, la gestión de recursos humanos, los procesos democráticos internos y el cuidado del medio ambiente. Aquí, la idea es de lograr un comportamiento organizacional éticamente ejemplar para la educación continua no formal (desde los mismos hábitos cotidianos rutinarios) de todos los integrantes de la Universidad, trabajadores administrativos, personal docente, estudiantes.

2- Formación ciudadana y profesional responsable: gestión socialmente responsable de la formación académica y pedagógica, tanto en sus temáticas, organización curricular como en sus metodologías y propuestas didácticas. La idea es que la formación profesional y humanística se oriente realmente hacia un perfil del egresado que haya logrado e incorporado competencias ciudadanas de Responsabilidad Social para el desarrollo sostenible de su sociedad. Esto implica que la orientación curricular general, y parte de los cursos de cada carrera, tengan una relación estrecha con los problemas reales de desarrollo y puedan dictarse en contacto directo con actores externos, bajo el método del aprendizaje más servicio.

3- Gestión social del conocimiento: gestión socialmente responsable de la producción y difusión del saber, la investigación, y los modelos epistemológicos promovidos desde el aula; para orientar la actividad científica y la práctica experta hacia su responsabilización social; no sólo a través de una negociación de las líneas de investigación universitaria con interlocutores externos; sino también para que los procesos de construcción de los conocimientos se den de modo participativo, con actores no académicos.

4- Comunidades de aprendizaje mutuo para el desarrollo: gestión socialmente responsable de la participación social de la Universidad en el desarrollo humano sostenible de la comunidad. La intención es organizar proyectos con actores externos de tal modo que se constituyan vínculos de puente (capital social) para el desarrollo social entre los participantes de los proyectos, de modo que estos últimos puedan aprender juntos (tanto los participantes académicos como los no académicos) durante el intercambio. Razón por la cual la participación socialmente responsable de la Universidad en el desarrollo social de su entorno no se limita en proyección para la capacitación de diversos públicos necesitados, sino que promueve la constitución de comunidades de aprendizaje mutuo para el desarrollo, en las que se beneficie tanto los estudiantes y los docentes como los actores externos.

La Responsabilidad Social de la Universitaria Nacional del Nordeste debe ser desarrollada con una política de gestión de calidad ética que busque alinear sus cuatro procesos (gestión, docencia, investigación, extensión) con la misión universitaria, sus valores y compromiso social, mediante el logro de la congruencia institucional, la transparencia y la participación dialógica de toda la comunidad universitaria (autoridades, estudiantes, docentes, adminis- 
trativos) con los múltiples actores sociales interesados en su buen desempeño universitario y necesitados de él, para la transformación efectiva de la sociedad hacia la solución de sus problemas de exclusión, inequidad, y sostenibilidad.

La congruencia institucional se logra a través de la alineación de los cuatro procesos universitarios con la misión, así como la vigilancia permanente de que los efectos directos y colaterales de la actividad universitaria no entren en contradicción con los fines pregonados, sino que vayan más bien realizando y reforzándolos. Para ello, se necesita involucrar a los actores de la comunidad universitaria en un auto-diagnóstico continuo del quehacer institucional, con herramientas apropiadas para garantizar la transparencia, la participación, y la mejora continua de nuestra Universidad hacia su responsabilidad social, enmarcada en la búsqueda global de un desarrollo más humano y más sostenible.

\section{CONCLUSION}

La Responsabilidad Social de la Universidad nos permite transitar en la búsqueda constante de redefinición y crítica permanente acerca de la manera de pensar y abordar los problemas educativos. Nos incita a desafíos para comprender los procesos cambiantes del mundo actual, y en esa medida ir modificando las formas de producción de conocimiento como fundamento para la formación de capital social.

La Responsabilidad Social de la Universidad Nacional del Nordeste es una plataforma para pensar que la ética, los valores de solidaridad, las relaciones sociales, la confianza recíproca, la reciprocidad y la cooperación son productoras de una riqueza y beneficio social sostenible y pueden ser un excelente negocio para la sociedad.

La Universidad debe asumir que la responsabilidad social sea el eje de las estrategias formativas del estudiante; en su formación integral y de ciudadano responsable como futuro profesional involucrado en los proyectos de su comunidad. Así también, debe servir como instancia crítica permanente que fortalezca las acciones de todas sus funciones: académica, investigación, extensión y como un modelo de gestión institucional.

Para ello es fundamental una integración curricular que responda a esta lógica de la Responsabilidad Social mediante la elección de modelos educativos adecuados y dentro de ellos insertar armónicamente la formación ética y en Responsabilidad Social.

Es preciso enfocar, ayudar y exigir a nuestros profesores y estudiantes que se empeñen en orientar las tesis de grado, monografías y demás actividades investigativas, a los problemas más urgentes del país y la región. Las tesis de nuestros estudiantes no pueden seguir sobrecargando los anaqueles de nuestras bibliotecas o los archivos de nuestras computadoras; han de hacerse reales y encontrar el mejor lugar de conservación en las estructuras mismas de nuestra sociedad.

La Universidad del sol es la esperanza de la sociedad y especialmente de los jóvenes; como coprotagonista junto al Estado y a otros sectores; para lograr la sociedad soñada; generando las transformaciones necesarias que a través de sus rayos iluminados de ética; proyectan el camino a la realización plena del ser humano. 


\section{REFERENCIAS BIBLIOGRAFICAS}

Cohen, Daniel: "Responsabilidad Social: Desafíos de la Universidad”. Revista DirCom, No 65, 2006. Buenos Aires. Noviembre de 2006.

De la Isla, Carlos; “Responsabilidad Social y Universidad”-México 1998.

Fereira, Emilio, Ética y Valores en la nueva PDVSA, (presentación), Venezuela, 2005. "Hacia la creación de Sistemas de Gestión Ética”. Conferencia celebrada en Madrid, 28/11/2005.

Kliksberg Bernardo; Disertación acto de entrega del Doctorado Honoris Causa de la Universidad Rey Juan Carlos de España. "Los desafíos éticos pendientes en un mundo paradojal; el rol de la Universidad", 2008.

Kliksberg,Bernardo; “La Responsabilidad Social Universitaria” Programa PNUD, Buenos Aires 2.005.

Nava Melin "Innovación para el desarrollo. La responsabilidad social de la Universidad venezolana en el presente” Foro Venezuela Institucionalidad, Democracia y Desarrollo.

http://www.cee.usb.ve/foro venezuela.htm.

Torres Pernalete Mariela y Trápaga Ortega;"Responsabilidad social de la Universidad"Retos y Perspectivas-Paidós- 2010.

Vallaeys François; Responsabilidad Social Universitaria "Propuesta para una definición madura y eficiente” Tecnológico de Monterrey,N.L. México. 2007.

Villar Javier; Responsabilidad Social Universitaria: nuevos paradigmas para una educación liberadora y humanizadora de las personas y las sociedades. Centro de Ética y Responsabilidad Social Juan Pablo II de la Universidad Católica de Temuco (Chile).

UNESCO "Conferencia Mundial sobre Educación Superior" Debate Temático sobre la Responsabilidad Social, la autonomía y la libertad académica de las universidades.

(1998), “Declaración mundial sobre educación superior en el siglo XXI; visión y acción”., en Conferencia mundial sobre educación superior ,Paris.

"Universidad construye país", http://www.construyepais.cl 


\section{CURRÍCULUM VITAE}

HECTOR LUIS OLIVA.

Contador Público, egresado de la Facultad de Ciencias Económicas-UNNE (1984).

Profesor en Ciencias Económicas, Facultad de Humanidades-UNNE (2010).

Coordinador del Fondo Compensador- ISUNNE (d.2012).

Secretario de Extensión Universitaria de la UTN- Resistencia (2000-2003).

Prof. Adjunto por concurso de Administración Financiera en la carrera Lic. en Administración Rural -UTN- Resistencia (d.1999).

Auxiliar de 1ra. por concurso en Finanzas de Empresas, Facultad de Ciencias EconómicasUNNE (d.1987).

Auxiliar de primera en la cátedra de Matemática Financiera - Facultad de Ciencias Económicas- UNNE (1989-1999) (d.2010).

hecoliva@yahoo.com.ar 